Document downloaded from:

http://hdl.handle.net/10251/58430

This paper must be cited as:

Capmany Francoy, J.; Gasulla Mestre, I.; Sales Maicas, S. (2011). Microwave photonics: Harnessing slow light. Nature Photonics. 5(12):731-733. doi:10.1038/nphoton.2011.290.

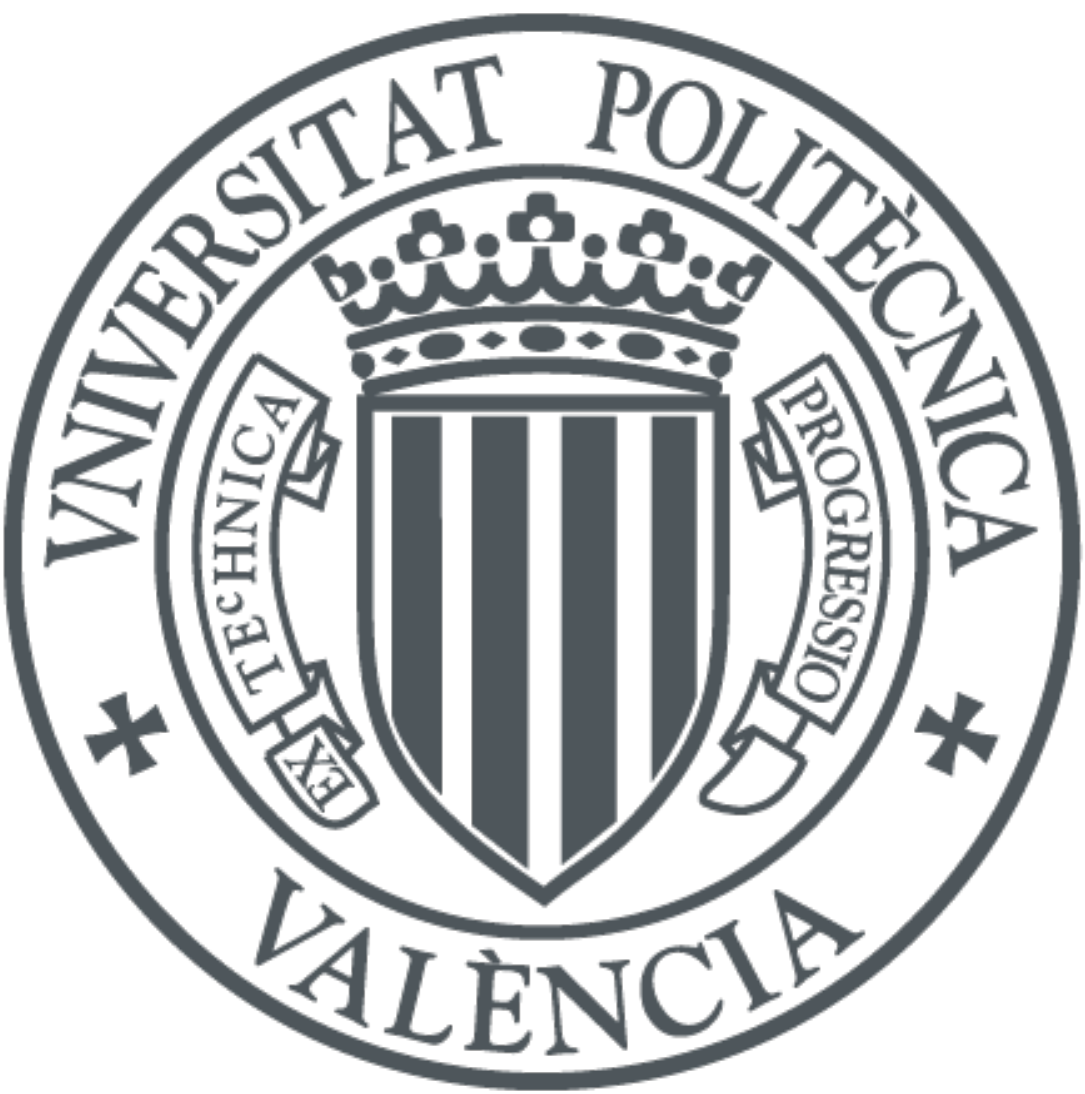

The final publication is available at

http://dx.doi.org/10.1038/nphoton.2011.290

Copyright Nature Publishing Group

Additional Information 


\title{
Harnessing slow light
}

\author{
José Capmany, Ivana Gasulla and Salvador Sales
}

Slow light techniques originally conceived for buffering high-speed digital optical signals now look set to play an important role in providing broadband phase and true time delays for microwave signals.

For the past 25 years, microwave photonic (MWP) systems and links have relied almost exclusively on discrete optoelectronic devices (semiconductor lasers and pin photodetectors) and standard optical fibres and fibre components (couplers, isolators, power dividers and multiplexers). These technologies have be used to support wellknown traditional radio transmission techniques such as linearization, mixing, intermediate frequency up- and down-conversion and subcarrier multiplexing.

However, the deployment of converged access networks- which marry radio and optical networking- would greatly benefit from the development of new type of microwave photonic technologies that offer greater functionality and chip-scale integration. Two approaches to tackling this task -slow and fast light (SFL) techniqueshave made considerable process in recent years.

SFL techniques aim to control the group velocity of an optical signal propagating in a particular device or medium. The propagation of a time-varying light signal through a dispersive optical medium implies that each of its frequency components will travel with a different phase velocity, resulting in an overall envelope time shift that is inversely proportional to the group index of the material $n_{g}$, which in turn depends on the dispersion of the refractive index $\mathrm{d} n / \mathrm{d} \omega$. A slow light medium is one that exhibits normal dispersion, $v_{g}<<c$, which is achieved by forcing $\mathrm{d} n / \mathrm{d} \omega$ to be large and positive. In contrary, a fast light medium is one that exhibits anomalous dispersion $P$.

Several nonlinear effects have been proposed as techniques to implement SFL devices including electromagnetic induced transparency in cold atoms, stimulated Brillouin scattering and stimulated Raman scattering in optical fibres, Coherent Population Oscillation in semiconductor waveguides, photonic crystals, dispersioncompensating fibres, fibre Bragg gratings and coupled cavities. All of these schemes can be characterized by the same common feature: the existence of either a single or multiple resonances. An absorption or gain resonance can be provided by a simple atomic transition or, in the case of a microresonator or Bragg grating, using nonlinear processes such as resonant scattering, spectral hole burning or four-wave mixing.

Originally conceived for buffering high-speed digital optical signals, SFL techniques are now being employed to provide broadband phase delays and true time delays for microwave signals. Tunable control over phase delays is essential for the implementation of broadband MWP reconfigurable filters so that varying the resonance position does not affect the filter spectral period or the width of the bandpass filter. Tunable control over true time delay is instrumental to the implementation of broadband phase array systems, which find applications in radar and satellite communications. Figure 1 helps to explain the differences between these two key functionalities. 


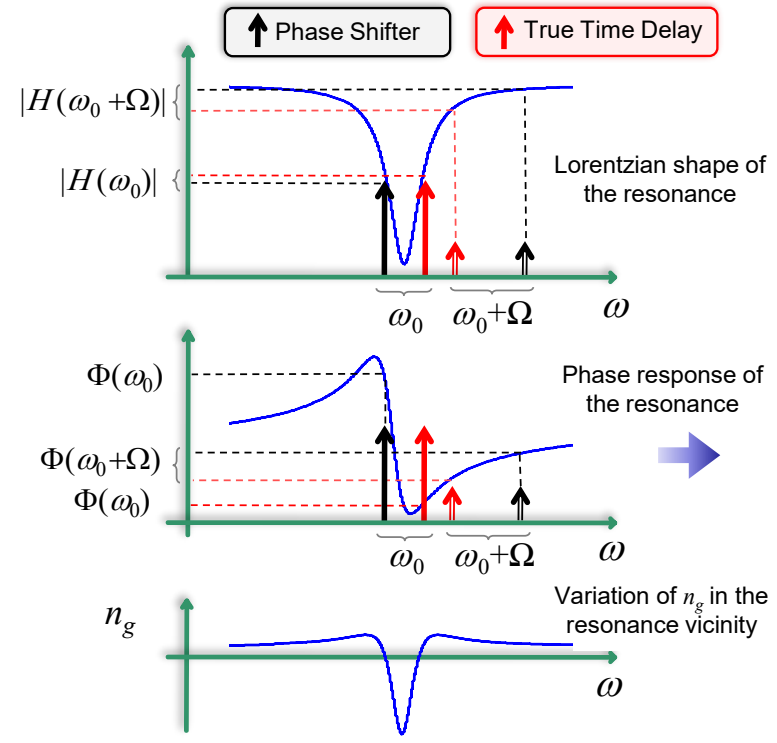

(a)

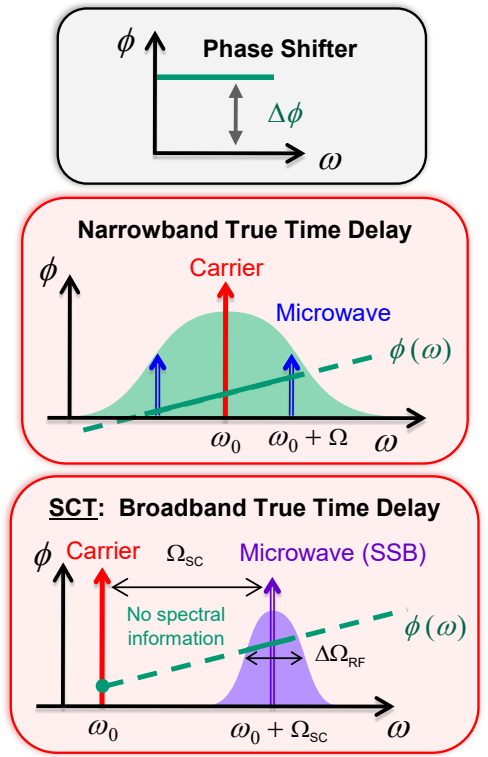

(b)

Figure 1: a-c, a-c, The Lorentzian amplitude (a) and phase (b) responses that characterize a photonic resonant medium give rise to a large variation in the material's group index $n_{g}$ (c). Implementing a microwave phase shifter (black arrow) or a true time delay (red arrow) requires the optical carrier and the radiofrequency subcarrier to be in different spectral locations. d, Phase shift response of the resonance; narrowband true time delay. e, The separate carrier tuning technique; broadband true time delay.

Imagine, for instance, an optical signal traversing a photonic resonant medium, such as a coupled resonator optical waveguide exhibiting an amplitude dip or resonance. A tunable radiofrequency phase shift can be achieved if we spectrally place this signal such that the optical carrier falls inside the resonance while the radiofrequency subcarrier stays outside. When beating occurs at the receiver, the detected signal will experience a phase shift that is determined by the phase difference between the optical carrier and the subcarrier. On the other hand, implementing a tunable true time delay requires both the carrier and the modulating sideband to be located inside the resonance. In both schemes, a variation in the spectral position of the optical carrier provides the desired tunability of either the phase shift or the delay imposed upon the detected radiofrequency signal.

Unfortunately, achieving a true time delay suffers from the same limitation experienced when buffering digital optical signals; SFL systems have a trade-off between the achievable time delay and bandwidth. In the case of MWP systems, an elegant solution known as separate carrier tuning was proposed by Paul Morton and Jacob Khurgin from Johns Hopkins University, Maryland, USA. This technique, which exploits the fact that most of the spectral region between the carrier and the sideband is unoccupied, requires a constant group delay in the subcarrier region as long as an appropriate phase shift is also imposed on the carrier.

Researchers have successfully demonstrated the separate carrier tuning technique by using stimulated Brillouin scattering in fibres to produce a tunable complex-valued two-tap filter featuring a delay line with a bandwidth of $120 \mathrm{MHz}$ at a central frequency of $6 \mathrm{GHz}$.

The European project GOSPEL (Governing the Speed of Light) has had notable success in developing a suite of MWP devices. In relation to broadband phase shifters 
for microwave signals, the use of coherent population oscillations in a semiconductor optical amplifier offers a bandwidth of several tens of gigahertzs while providing small size, low weight and also the possibility of on-chip integration. Tunability has been demonstrated either by optical pumping or changing the electrical injection current. Researchers from the Universitat Politècnica de València (UPVLC), Spain, and the Danish Technical University (DTU) have demonstrated phase shifts up to $360^{\circ}$ over bandwidths exceeding $40 \mathrm{GHz}$ by cascading five SOAs stages followed by three intermediate optical filters. This configuration has recently been refined to realize a full $360^{\circ}$ tunable microwave phase shifter based on a single semiconductor optical amplifier.

Researchers at DTU have also demonstrated a tunable microwave phase shifter based on a silicon-on-insulator dual-microring resonator. The device provides a quasi-linear phase shift of up to $360^{\circ}$ with a radiofrequency power variation lower than $2 \mathrm{~dB}$ and a frequency bandwidth of $40 \mathrm{GHz}$. Collaborations from DTU and UPVLC have also demonstrated a tunable MWP filter in the $20 \mathrm{GHz}$ region based on a single silicon-on-insulator microring resonator phase shifter.

Stimulated Brillouin scattering in fibres can be exploit to realize a less compact microwave phase shifter that has the advantage of great bandwidth and easier tuning. The first scheme proposed by researchers at the Universidad Pública de Navarra (UPNA) in Spain, featured bandwidths of $20 \mathrm{GHz}$. In a recent collaboration between UPNA, UPVLC and the École Polytechnique Fédérale de Lausanne (EPFL), researchers extended the achievable bandwidth to over $50 \mathrm{GHz}$, which demonstrates potential for reaching the millimetre-wavelength region.

Several SFL-based approaches have been reported for implementing true time delays for microwave signals using photonic devices, including those based on stimulated Brillouin scattering and stimulated Raman scattering in optical fibres, coherent population oscillations in erbium-doped fibre amplifiers, EIT in gas-filled hollow-core fibres, and by employing wavelength conversion and fibre dispersion. Microring resonators have been attracting significant attention because of their compact size and opportunity for chip-scale integration. Although in isolation microring resonators provide only short delays over small bandwidths, larger bandwidths and larger delays can be achieved by placing several slightly detuned rings in a cascade, as proposed by Khurgin. Dutch firm LioniX and researchers at the University of Twente have recently used this technique to create an optical beamforming network.

Photonic crystal waveguides based on line defects can also be used to implement true time delays. Recent work by researchers at St. Andrews University in the UK shows that photonic crystal waveguides offer better figure of merits than microring resonators at very high frequencies. French firm Thales has fabricated the first waveguides for this purpose and has also collaborated with UPVLC to develop the first implementation of MWP filters. Researchers at university of Kassel in Germany, Technion University in Israel and DTU expect further flexibility by incorporating quantum dots to increase the delay and tunability of photonic crystal waveguides. In addition, researchers at the Centre for Ultrahigh Bandwidth Devices for Optical Systems in Australia are currently working to generate long delays by enhancing nonlinear effects in waveguides.

Scientists have also demonstrated the integration of different MWP functionalities. Researchers at the University of Twente and LioniX recently implemented an integrated MWP filter consisting of a reconfigurable optical delay line onto a single CMOS-compatible photonic chip. A complex multicavity design (Fig. 2a,b) reported by researchers from Kotura and Telcordia features 1-2 GHz bandwidth filters 
with very high extinction ratios $(\sim 50 \mathrm{~dB})$. The silicon waveguides employed to construct these filters have propagation losses of just $\sim 0.5 \mathrm{~dB} / \mathrm{cm}$. Each cavity is thermally controlled by metal heaters and placed on the top of the ring. With a power dissipation of around $72 \mathrm{~mW}$, the ring resonance can be tuned by one free spectral range, thus resulting in wavelength-tunable filters. Both second-order and fifth-order ring resonators have been implemented so far.
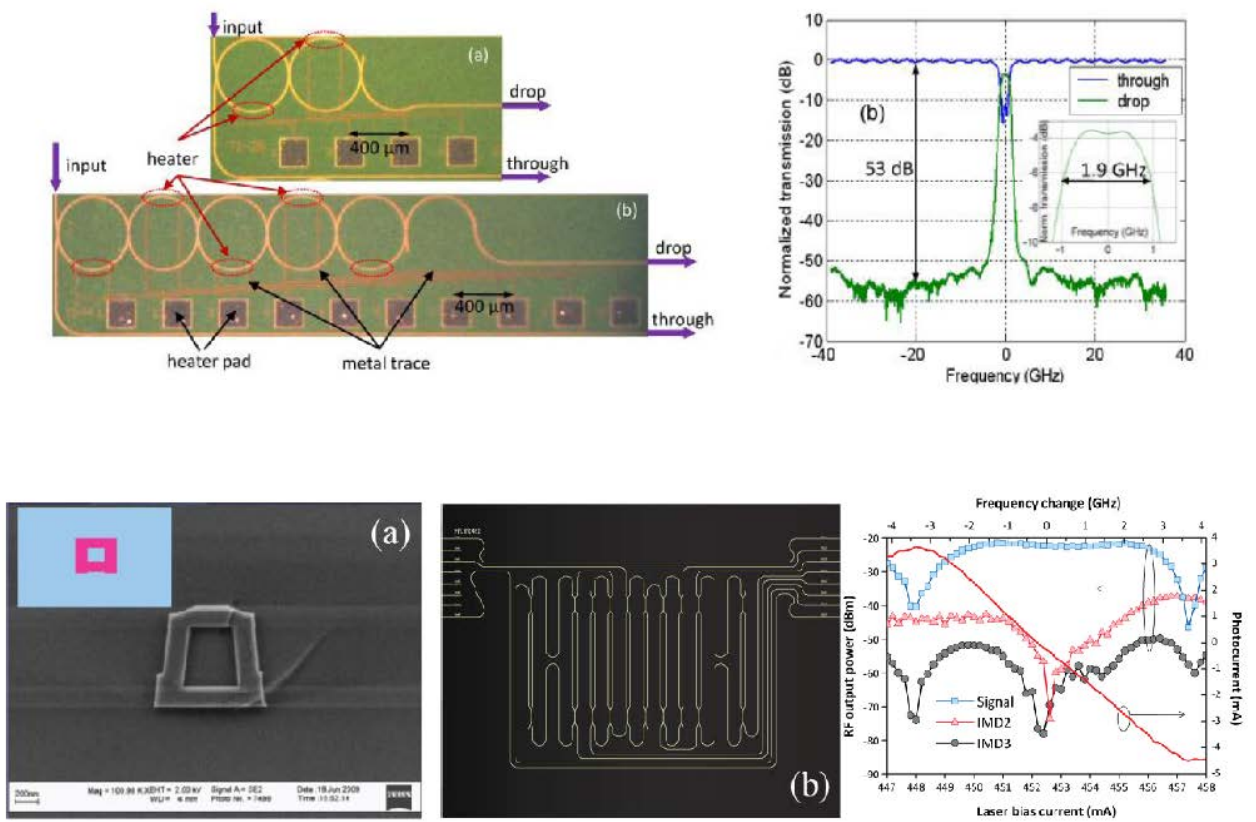

Figure 2. a,b, Second- and fifth-order gigahertz-bandwidth optical filters based on high-order silicon ring resonators developed by Kotura and Telcordia (a) with measured transfer functions (b). c-e, Photonics-integrated discriminator for phase-modulated MWP links implemented by researchers at the University of Twente and LioniX. c, Scanning electron microscope image of the TriPleX waveguide configuration. d, Integrated filter layout. e, Measured discriminator transfer function.

In the field of signal transmission and conditioning, researchers from University of Twente and LioniX demonstrated the first integrated discriminator for phasemodulated MWP links. The photonic chip in this work consists of five optical ring resonators that could be controlled through thermo-optical tuning. The layout, shown in Fig. 2c-e, is based on waveguides implemented using the LioniX's high-contrast TripleX technology.

These examples indicate that the realization of integrated chips featuring sophisticated MWP circuitry is no longer a distant dream, but will soon become a reality.

José Capmany, Ivana Gasulla and Salvador Sales are researchers at the Optical and Quantum Communications Group, Institute of Telecommunications and Multimedia Applications, Universitat Politècnica de València, Camino de Vera s/n, 46022 Valencia, Spain.

e-mail: jcapmany@dcom.upv.es 